\title{
Long-Term Side Effects of Adjuvant Therapy in Primary Breast Cancer Patients: Results of a Web-Based Survey
}

\author{
Renate Haidinger ${ }^{\mathrm{a}}$ Ingo Bauerfeind ${ }^{\mathrm{b}}$ \\ ${ }^{a}$ Brustkrebs Deutschland e.V./German Breast Cancer Association, Hohenbrunn, ${ }^{\mathrm{b}}$ Breast Center, Department of \\ Obstetrics and Gynecology, Landshut Hospital, Landshut, Germany
}

\section{Keywords}

Adjuvant treatment $\cdot$ Aromatase inhibitors · Breast cancer - Chemotherapy - Endocrine therapy - Quality of life . Side effects $\cdot$ Toxicity

\begin{abstract}
Background: Chronic treatment sequelae may substantially reduce the long-term quality of life in breast cancer survivors. Methods: We report a comprehensive Web-based survey on the presence of long-term side effects of adjuvant anti-breast cancer therapy in 1,506 patients who had been diagnosed with primary breast cancer at least 1 year before. Results: Fatigue, depression, depressive mood, concentration deficit, pain, changes of mucosa and skin appendages, as well as symptoms of peripheral neuropathy were the most prevalent reported complaints. Chemotherapies - taxanebased regimens in particular - were associated with increased rates of long-term symptoms, including persistent peripheral neuropathy. Overall, the data show a substantial prevalence of a wide variety of potentially treatment-associated symptoms over a protracted time frame after the diagnosis of breast cancer. The burden of symptoms was high for fatigue, depression, sleep disturbances, pain, and peripheral neuropathic symptoms. Conclusion: Estimating the burden of chronic toxicities should contribute to enhance rational decision-making on treatments including chemotherapy in patients with low versus high risk of recurrence.
\end{abstract}

() 2019 S. Karger AG, Basel

\section{Introduction}

Almost all patients with primary breast cancer receive adjuvant cancer therapies to reduce the risk of local recurrence and metastases. While these antihormonal and chemotherapeutic treatments help to improve long-term disease-free survival and/or overall survival in a significant proportion of patients, these therapies are associated with significant acute and chronic toxicities [1-3]. Chronic treatment sequelae may substantially reduce the longterm quality of life in large numbers of breast cancer survivors. Estimating the burden of these chronic toxicities is an important component of a comprehensive evaluation of patient-reported outcomes. It may thus contribute to enhance rational decision-making on the use of chemotherapies in patients with low- versus high-risk breast cancer.

\section{Methods}

We performed a comprehensive survey on long-term side effects of adjuvant therapy in patients who had previously been diagnosed with primary breast cancer at least 1 year before taking the survey. An electronic questionnaire was provided during May and June 2016 on the Facebook social network page [4] and the website of Brustkrebs Deutschland e.V. [5], a German breast cancer organization.

Data gathered included demographic characteristics, time interval since diagnosis, histological type, size and spread of breast cancer, receptor and lymph node status, type of adjuvant therapies and the specific drugs used, metastasis status, and secondary malignancies. Patients provided information on the presence of longterm side effects by checking listings of potential side effect plus optional free-text entry. They also rated the side effects by their

\section{KARGER}

(c) 2019 S. Karger AG, Basel 
Table 1. Demographic, disease, and anticancer treatment characteristics

\begin{tabular}{|c|c|}
\hline Parameter & $\begin{array}{l}\text { Value } \\
(\mathrm{N}=1,506)\end{array}$ \\
\hline \multicolumn{2}{|l|}{ Age } \\
\hline Median, years (range) & $49(20-81)$ \\
\hline$<35$ years, $\%$ & 7 \\
\hline$>35-45$ years, $\%$ & 24 \\
\hline$>45-55$ years, $\%$ & 43 \\
\hline$>55-65$ years, $\%$ & 20 \\
\hline$>65$ years, $\%$ & 6 \\
\hline \multicolumn{2}{|c|}{ Time since primary diagnosis of breast cancer, $\%$} \\
\hline $1-2$ years & 39 \\
\hline $3-5$ years & 30 \\
\hline $6-10$ years & 21 \\
\hline$\geq 11$ years & 10 \\
\hline \multicolumn{2}{|l|}{ Breast cancer characteristics, $\%$} \\
\hline HR(ER and/or PR)-positive & 56 \\
\hline HER2 $0,1+$, or $2+$ & 19 \\
\hline HER2-positive 3+ & 15 \\
\hline Triple-negative & 17 \\
\hline Sentinel lymph node involvement & 31 \\
\hline Additional lymph node involvement & 27 \\
\hline \multicolumn{2}{|l|}{ Tumor stage, $\%$} \\
\hline $\mathrm{T} 1$ & 45 \\
\hline $\mathrm{T} 2$ & 45 \\
\hline T3 & 9 \\
\hline $\mathrm{T} 4$ & 2 \\
\hline \multicolumn{2}{|l|}{ Metastatic disease, $\%$} \\
\hline Metachronous metastases & 4 \\
\hline \multicolumn{2}{|l|}{ Anticancer therapy, \% } \\
\hline Radiotherapy & 82 \\
\hline Antihormonal therapy & 78 \\
\hline Chemotherapy & 75 \\
\hline HER2-targeted therapies ${ }^{\mathrm{a}}$ & 26 \\
\hline
\end{tabular}

apatients could have received 1 or more HER2-targeted monoclonal antibodies.

$\mathrm{HR}$, hormone receptor; ER, estrogen receptor; $\mathrm{PR}$, progesterone receptor; HER2, human epidermal growth factor receptor 2.

respective frequency, and thus the periods with perceived severe burden, in five categories.

Data from patients who completed all relevant questions were compiled using spreadsheet software and analyzed using descriptive statistics from Survey Monkey (Web-based survey tool).

\section{Results}

\section{Demographic Data}

A total of 1,506 patients fully participated in the survey. Their median age was 49 years (range $20-81$ years); $43 \%$ of the patients were $45-55$ years old and $99 \%$ were female. The median time since breast cancer diagnosis was in the range of $3-5$ years; $31 \%$ of the patients had received their diagnosis more than 5 years before taking the survey (table 1).
Table 2. Drugs used for anticancer treatment

\begin{tabular}{lr}
\hline Parameter & Value $(\mathrm{N}=1,506)$ \\
\hline Chemotherapies, \% & \\
$\quad$ Epirubicin & 44 \\
Cyclophosphamide & 37 \\
Paclitaxel & 32 \\
Docetaxel & 26 \\
Carboplatin & 8 \\
Fluorouracil & 8 \\
Doxorubicin & 7 \\
nab-Paclitaxel & 3 \\
Liposomal doxorubicin & 1 \\
No data & 8 \\
No chemotherapy & 26 \\
Antihormonal drugs, \% & \\
Tamoxifen & 80 \\
Aromatase inhibitor & 32 \\
Aromatase inhibitor and tamoxifen & 12 \\
GnRH analogs & 5 \\
Antibodies, \% & \\
Trastuzumab & 18 \\
Pertuzumab & 1 \\
Others, \% & 0.2 \\
Trastuzumab-emtasine & 1 \\
Lapatinib & \\
\hline GnRH, gonatropin-releasing hormone. & \\
\hline
\end{tabular}

\section{Disease Characteristics}

In terms of the receptor status, $56 \%$ of the patients reported a hormone receptor-positive breast cancer, 15\% indicated a human epidermal growth factor receptor 2(HER2)-positive (3+) status, and $17 \%$, a triple-negative tumor (defined as negative for estrogen and progesterone receptors AND not HER2 3+; any reallocations of HER2 2+ patients by in situ hybridization were not accounted for). The others did not report on their receptor status.

A positive sentinel lymph node was reported by $31 \%$ of the patients, and $27 \%$ indicated further involved lymph nodes. Tumor size was $<2 \mathrm{~cm}$ (T1) in $45 \%, 2-5 \mathrm{~cm}$ (T2) in $45 \%$, and $>5 \mathrm{~cm}(\mathrm{~T} 3 / \mathrm{T} 4)$ in $9 \%$ of the patients. Since the primary breast cancer diagnosis, metastatic disease had been detected in $4 \%$ of the patients, mostly affecting bones, lymph nodes, and lungs; $10 \%$ did not report their metastatic status.

\section{Adjuvant Breast Cancer Treatments}

Radiotherapy (82\%), antihormonal treatment (78\%), and chemotherapy (75\%) were the most common modalities of adjuvant anticancer therapy. There were some inconsistencies in the reports on antihormonal therapies: $16 \%$ of the patients reported an antihormonal therapy despite having stated an absence of hormone receptor positivity; $6 \%$ received an antihormonal drug despite having 
reported no antihormonal treatment. Therefore, a total of $78 \%$ probably had a hormone receptor-positive breast cancer.

Taxanes (61\%), anthracyclines (52\%), and cyclophosphamide (37\%) were the most commonly used chemotherapeutics.

A total of $18 \%$ of all patients reported to have received HER2-directed therapies; this includes $80 \%$ of the patients with HER $23+$ positive status and $24 \%$ of those with HER2 0-2+ status.

In the subgroup of patients treated with antihormonal drugs, tamoxifen (80\%) and aromatase inhibitors (32\%) were the most commonly used substances (table 2 ).

\section{Information on Potential Side Effects}

Patients received information on potential side effects of adjuvant cancer therapy predominantly from their treating physician (81\%), the Internet (32\%), information brochures (27\%), and nursing staff (27\%).

\section{Long-Term Side Effects of Adjuvant Therapies}

Regardless of the type of adjuvant therapy, hot flashes, fatigue, and sleep disturbances were the most common symptoms, each reported by more than two-thirds of the patients. Pain in bones, muscles, or joints was also highly prevalent, each reported by at least half of the overall patient cohort. A similar proportion of patients experienced cognitive and mood disturbances (concentration deficit, symptoms of depression). Peripheral neuropathic symptoms including numbness and/or tingling of feet or hands was documented by a quarter of the cohort. Hair and nails were affected in more than one-third of the patients.

\section{Association of Chemotherapy with Long-Term Side Effects}

Overall, chemotherapy was associated with increased prevalence of all reported symptoms. Specifically, concentration deficits $(+22 \%)$, nail changes $(+31 \%)$, hair loss $(+33 \%)$, tingling of hands $(+20 \%)$, numbness $(+26 \%)$ and tingling of feet $(+22 \%)$ were increased by $\geq 20 \%$ versus adjuvant therapy without the use of antineoplastic chemotherapeutics (table 3, fig. 1).

Among all patients who reported taxane-containing therapies, the prevalences of neuropathic long-term effects such as numbness, tingling, and pain of hands or feet were substantially higher than in those who received no taxanes.

\section{Association of Taxane Chemotherapy with Long-Term Side Effects}

Compared to non-taxane-based adjuvant therapies, the use of taxanes was associated with a strongly increased prevalence of nail changes $(+26 \%)$, hair loss $(+22 \%)$, and
Table 3. Long-term side effects in patients without and with chemotherapy

\begin{tabular}{llll}
\hline & $\begin{array}{l}\text { Without chemo- } \begin{array}{l}\text { With chemo- } \\
\text { therapy, } \% \\
(N=384)\end{array} \\
\text { therapy, } \% \\
(N=1,122)\end{array}$ & $\begin{array}{l}\text { Difference, } \\
\%\end{array}$ \\
\hline Hair loss & 16 & 49 & 33 \\
Nail changes & 19 & 50 & 31 \\
Numbness, feet & 4 & 30 & 26 \\
Tingling, feet & 6 & 28 & 22 \\
Concentration deficit & 46 & 68 & 22 \\
Tingling, hands & 11 & 31 & 20 \\
Tingling and pain, feet & 8 & 24 & 16 \\
Numbness, hands & 13 & 29 & 16 \\
Bone pain & 50 & 63 & 13 \\
Tingling and pain, hands & 9 & 20 & 11 \\
Dry oral mucosa & 19 & 30 & 11 \\
Dry genital mucosa & 36 & 47 & 11 \\
Muscular pain & 41 & 52 & 11 \\
Loss of libido & 43 & 54 & 11 \\
Exhaustion & 61 & 72 & 11 \\
Vertigo & 17 & 25 & 8 \\
Joint pain & 58 & 65 & 7 \\
Dry eyes & 27 & 33 & 6 \\
Visual disturbances & 26 & 31 & 5 \\
Fatigue & 66 & 71 & 5 \\
Depressed mood & 43 & 46 & 3 \\
Depression & 14 & 16 & 2 \\
Sleep disturbances & 68 & 69 & 1 \\
Hot flashes & 71 & 72 & 1 \\
\hline
\end{tabular}

Table 4. Long-term side effects in patients who received adjuvant therapies without or with taxanes

\begin{tabular}{|c|c|c|c|}
\hline & $\begin{array}{l}\text { Adjuvant } \\
\text { therapy } \\
\text { without } \\
\text { taxanes, \% } \\
(N=652)\end{array}$ & $\begin{array}{l}\text { Adjuvant } \\
\text { therapy } \\
\text { including } \\
\text { taxanes, \% } \\
(N=854)\end{array}$ & $\begin{array}{l}\text { Difference, } \\
\%\end{array}$ \\
\hline Nail changes & 27 & 53 & 26 \\
\hline Numbness, feet & 10 & 34 & 24 \\
\hline Tingling, feet & 11 & 31 & 20 \\
\hline Concentration deficit & 52 & 71 & 19 \\
\hline Tingling, hands & 16 & 34 & 18 \\
\hline Numbness, hands & 16 & 31 & 15 \\
\hline Tingling and pain, feet & 12 & 26 & 14 \\
\hline Muscular pain & 42 & 54 & 12 \\
\hline Dry genital mucosa & 39 & 49 & 10 \\
\hline Tingling and pain, hands & 12 & 22 & 10 \\
\hline Exhaustion & 64 & 73 & 9 \\
\hline Bone pain & 54 & 63 & 9 \\
\hline Loss of libido & 46 & 55 & 9 \\
\hline Hot flashes & 67 & 75 & 8 \\
\hline Joint pain & 59 & 66 & 7 \\
\hline Visual disturbances & 25 & 32 & 7 \\
\hline Dry oral mucosa & 23 & 30 & 7 \\
\hline Fatigue & 67 & 72 & 5 \\
\hline Dry eyes & 29 & 34 & 5 \\
\hline Vertigo & 20 & 25 & 5 \\
\hline Sleep disturbances & 66 & 70 & 4 \\
\hline Hair loss/partial hair loss & 28 & 50 & 2 \\
\hline Depression/depressed mood & 52 & 52 & 0 \\
\hline
\end{tabular}




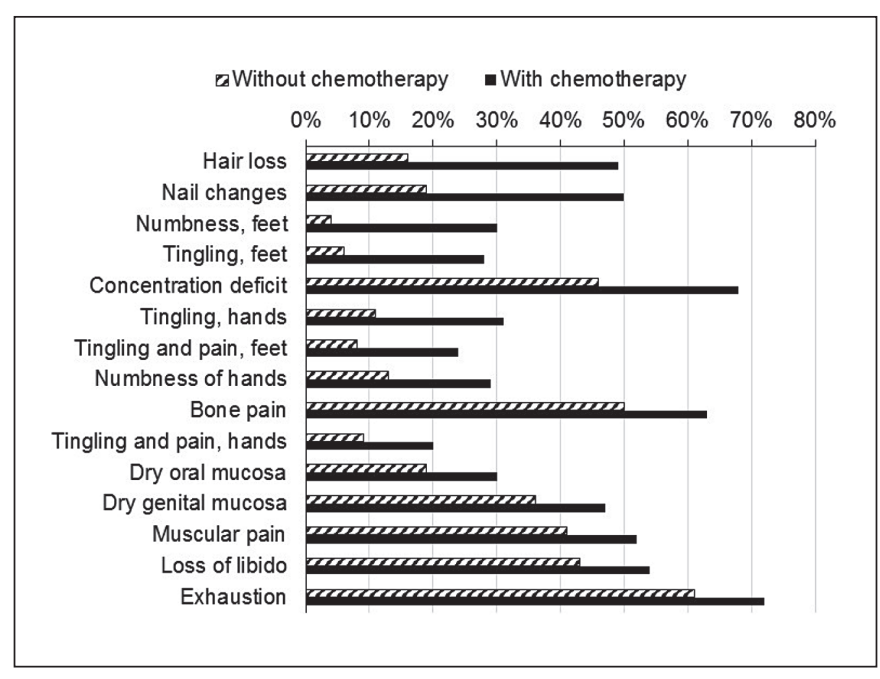

Fig. 1. Long-term prevalence (\%) of side effects in patients who received adjuvant therapies with or without the use of chemotherapeutic drugs (items with $\geq 10 \%$ difference in prevalence in both groups are shown in the order of decreasing difference).

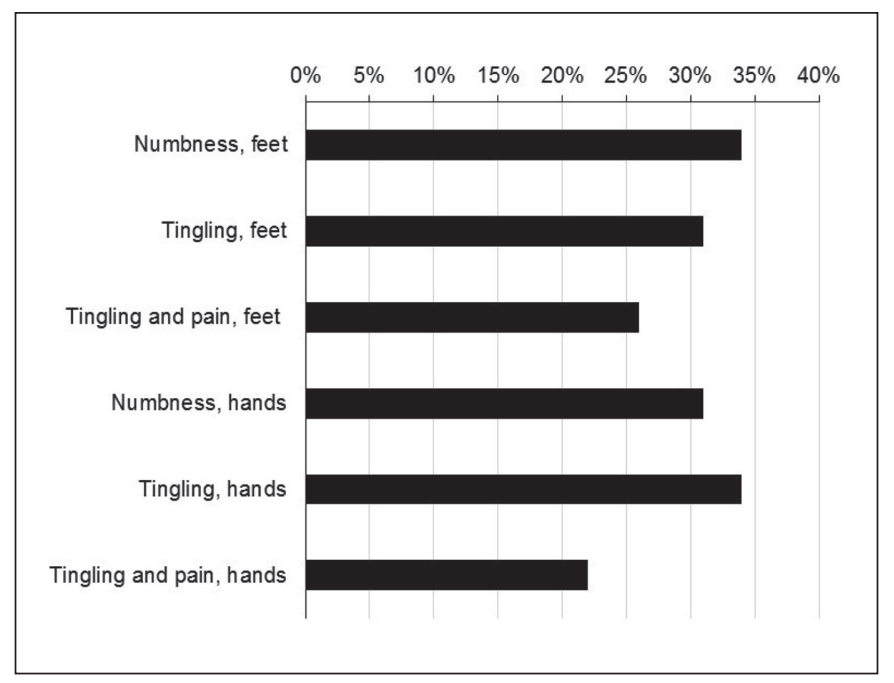

Fig. 3. Long-term prevalence (\%) of neuropathic side effects in patients who received adjuvant therapies with taxanes.

neuropathic symptoms: numbness $(+24 \%)$ and tingling $(+20 \%)$ of feet, numbness $(+15 \%)$ and tingling $(+18 \%)$ of hands, as well as tingling plus pain in feet $(+14 \%)$ and hands $(+10 \%)$ (table 4 , fig. 2 ). The neuropathic symptoms in taxane recipients (fig. 3) tended to be more prevalent in those with triple-negative versus receptor-positive tumors (fig. 4).

Association of Antihormonal Therapy with Long-

Term Side Effects

Compared to patients who did not receive endocrine therapy, those who used antihormonal drugs more often

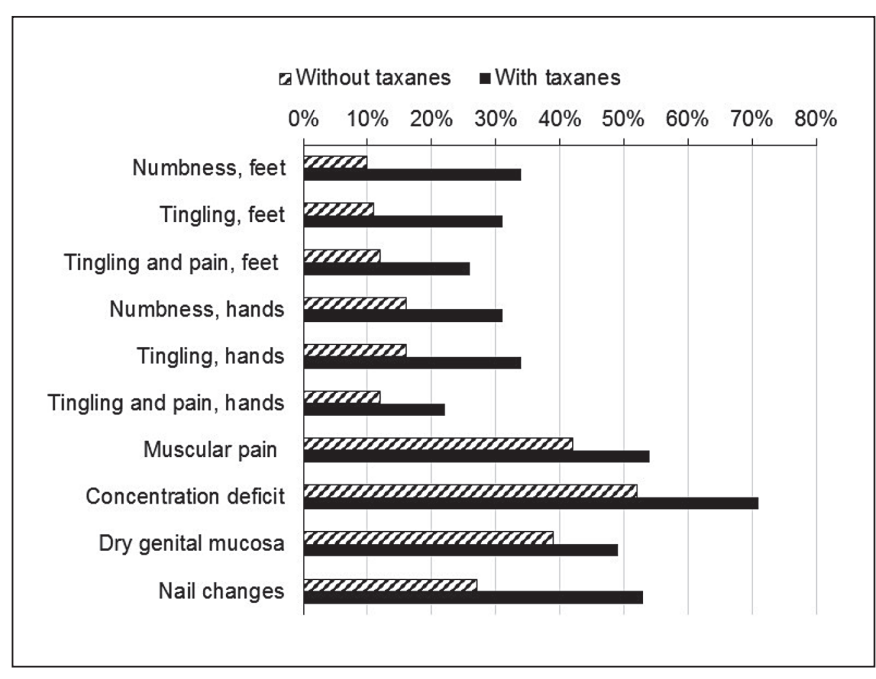

Fig. 2. Long-term prevalence (\%) of side effects in patients who received adjuvant therapies with or without the use of taxanes (items with $\geq 10 \%$ difference in prevalence in both groups).

reported hot flashes $(+19 \%)$ and vaginal dryness $(+11 \%)$, while hair loss was less common $(-19 \%)$. Dry eyes and visual disturbances were experienced by roughly onethird of patients taking these drugs.

Among the subclasses of antihormonal drugs, aromatase inhibitors were associated with a relatively high prevalence $(73 \%)$ of joint pain, whereas loss of libido was prevalent with gonadotropin-releasing hormone $(\mathrm{GnRH})$ analogs (69\%).

\section{Long-Term Side Effects in Patients with Exclusive}

Taxane Chemotherapy versus Exclusive Antihormonal Therapy

When comparing these two modalities, side effects were generally more prevalent in the taxane-treated patients. The association of certain symptoms, including concentration deficit $(+22 \%)$, nail changes $(+31 \%)$, hair loss $(+44 \%)$, and various peripheral neuropathy symptoms $(+12$ to $+25 \%$, particularly tingling and numbness of feet), with taxane therapy was apparent.

\section{Association of the Time Interval since Breast Cancer}

Diagnosis with Side Effects

The distribution of side effects was similar in patient groups with different time intervals since diagnosis. The side effects with $\geq 10 \%$ of difference of prevalence between two of these time frames were numbness of feet ( $17 \%$ at $>5$ years vs. $28 \%$ at $1-2$ years) and tingling of feet ( $17 \%$ at $>5$ years vs. $27 \%$ at $3-5$ years after diagnosis). 
Fig. 4. Long-term prevalence (\%) of side effects in patients who received adjuvant therapies without chemotherapeutics versus those treated with taxanes, shown by receptor status.

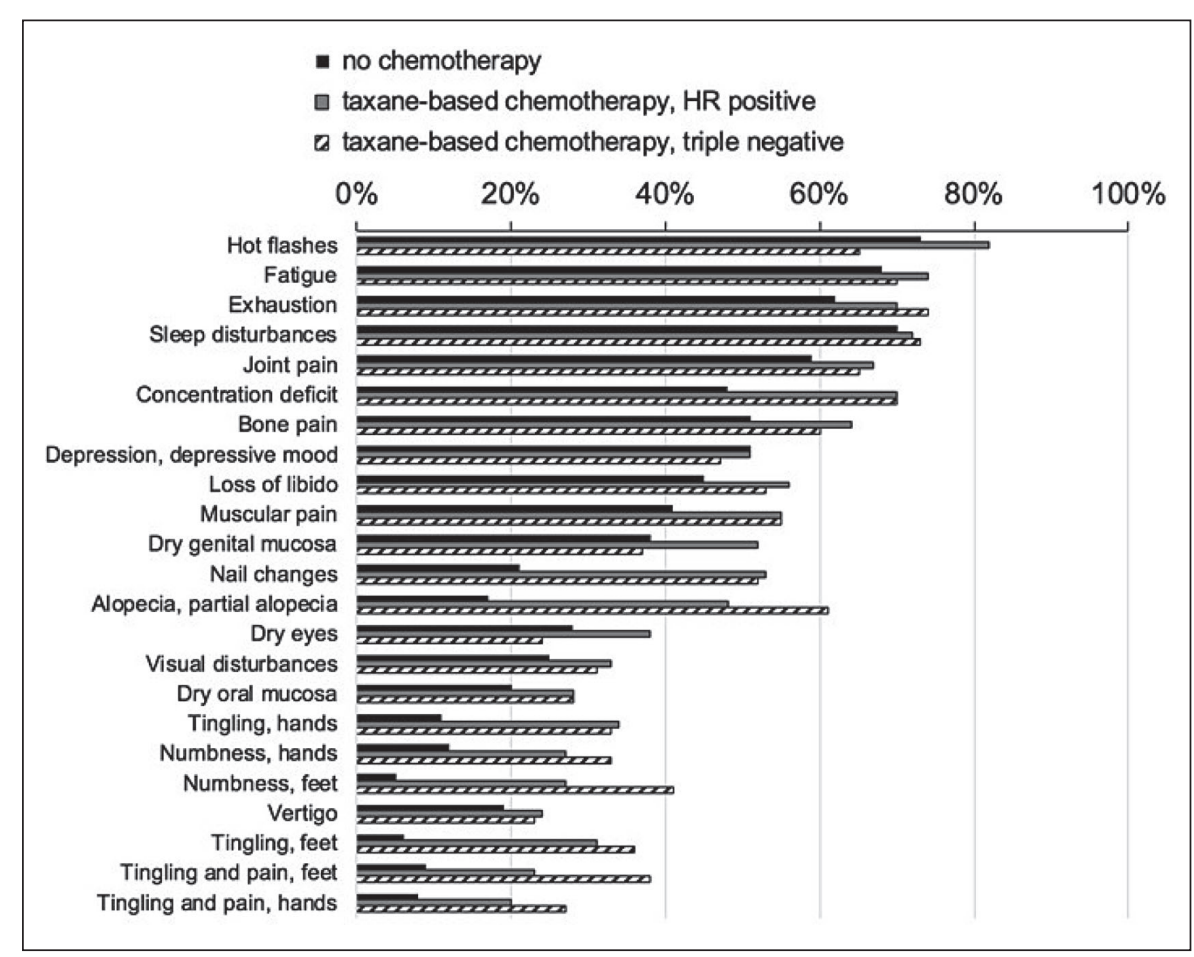

\section{Perceived Burden Associated with Side Effects}

The most severe burden of symptoms (categories always/often severely burdened) was reported for fatigue (79\%) and depression (79\%), sleep disturbances (77\%) and tiredness (76\%). Pain in various localizations (72$75 \%)$ caused an important burden as well. Peripheral neuropathic symptoms (numbness, tingling and pain in hands and feet) was reported as causing always/often a severe burden by $65-69 \%$ of the affected patients.

\section{Discussion}

We conducted a large Web-based survey in breast cancer patients with a broad range of characteristics and intervals after primary diagnosis. To our knowledge, this is the largest dataset on long-term sequelae of adjuvant breast cancer drug therapy in Germany.

Taxanes were the more frequently used antineoplastic drugs reflecting current treatment practice, while tamoxifen was used in most patients with antihormonal therapy.

Fatigue, depression/depressive mood, concentration deficit, pain, changes of mucosa and skin appendages, as well as symptoms of peripheral neuropathy were the most prevalent complaints. Overall, the data show a substantial prevalence of a wide variety of potentially therapy-associated symptoms over a protracted time frame after the diagnosis of breast cancer. The burden of symptoms, a measure of their severity and subjective effect, was high for many symptoms including fatigue, depression, sleep disturbances, and peripheral neuropathy. Of note, painful symptoms at various localizations were reported by a large proportion of the patients and showed higher longterm prevalence in the participants who received chemotherapy.

A striking result was the pronounced burden of persisting symptoms even in patients with a time since diagnosis of more than 5 years. In patients who received both antihormonal therapy and chemotherapy, the long-term persistent effects of chemotherapy may be superimposed with adverse effects of ongoing use of antihormonal drugs. It would therefore be of interest to analyze the time course of symptoms in patients who did not receive any antihormonal therapy (e.g., those with triple-negative breast cancer) to determine the prevalence of true lasting effects of chemotherapy in patients who have long since completed their adjuvant treatments. However, for certain symptoms that are characteristic of taxanes, such as peripheral neuropathy, the time course may well be deduced from the data: These symptoms show a somewhat declining but still substantial prevalence in patients with long time intervals of 5 years since diagnosis.

Our study is associated with several limitations. The use of an online questionnaire, particularly via Facebook, likely selected for younger patients, thus reducing the representativeness for the overall breast cancer population in Germany. Asking which (long-term) side effects occur without a clear specification of a time frame (e.g., the last 3 months) may have resulted in the inclusion of 
symptoms that had abated some time ago, thus blurring the distinction between slowly reversible and truly longterm symptoms for the patients with a short interval since diagnosis. However, the shortest interval since diagnosis included was 1 year, which makes it likely that symptoms present at this stage are actually chronic. In addition, the different intensities and durations of symptoms may result in recall biases. In the patients who developed metastases, the relapsed disease and its antineoplastic treatment might have caused symptoms unrelated to the initial adjuvant therapy.

Rating the burden of symptoms by the proportion of particularly burdensome episodes may result in an enhanced emphasis on rarely occurring severe symptoms versus permanently present but less intense symptoms. The true burden of symptoms may be more precisely described by measuring their frequency, duration, and intensity separately. A fraction of the patients did not report their hormone receptor status. We do not expect this to influence the reported side effect frequencies.

A number of symptoms, e.g., fatigue, depression, pain, and menopausal symptoms, may occur independently of any cancer therapy in the context of malignancies and ageing. In our cohort, roughly one-third of the patients were older than the mean age at menopause in the west- ern countries (51.4 years) [6]. These factors, which are unrelated to treatment, may have biased the estimates of frequencies and intensities of side effects.

\section{Conclusions}

Patient-reported outcomes have an increasingly important role in the assessment of quality of care in oncology. This analysis of a survey dataset acquired in a large population of breast cancer patients spans a broad range of follow-up durations. It indicates a high burden of longterm side effects of adjuvant anticancer therapy, particularly in patients who received taxane chemotherapeutics.

Assays that identify patients who are expected to have a long-term benefit from chemotherapy versus those who may not benefit may help to spare the use of toxic drugs in a significant percentage of patients, thus reducing the burden of long-term sequelae of chemotherapy. The ultimate effect may be improved quality of life in breast cancer survivors.

\section{Disclosure Statement}

Both authors have no conflict of interest to declare.

\section{References}

1 Miao H, Li J, Hu S, He X, Partridge SC, Ren J, Bian Y, Yu Y, Qiu B: Long-term cognitive impairment of breast cancer patients after chemotherapy: a functional MRI study. Eur J Radiol 2016;85:1053-1057.

$\checkmark 2$ Ejlertsen B: Adjuvant chemotherapy in early breast cancer. Dan Med J 2016;63:pii B5222.
Murtagh G, Lyons T, O’Connell E, Ballot J, Geraghty L, Fennelly D, Gullo G, Ledwidge M, Crown J, Gallagher J, Watson C, McDonald KM, Walshe JM: Late cardiac effects of chemotherapy in breast cancer survivors treated with adjuvant doxorubicin: 10-year follow-up. Breast Cancer Res Treat 2016;156:501-506.
4 https://de-de.facebook.com/brustkrebsd/.

5 http://brustkrebsdeutschland.de.

6 German Menopause Society, www.menopause-gesellschaft.de/mpg_privat/ 5302579a8e0cdf701/index. html (accessed July 4, 2017). 